\title{
Trapping neutral fermions with kink-like potentials
}

\author{
Antonio S. de Castro ${ }^{\mathrm{a}, \mathrm{b}}$, Marcelo Hott ${ }^{\mathrm{b}, *}$ \\ ${ }^{a}$ Universidade de Coimbra, Centro de Física Computacional, P-3004-516 Coimbra, Portugal \\ b UNESP, Campus de Guaratinguetá, Departamento de Física e Química, 12516-410 Guaratinguetá, SP, Brazil
}

Received 6 July 2005; received in revised form 11 November 2005; accepted 14 November 2005

Available online 21 November 2005

Communicated by P.R. Holland

\begin{abstract}
The intrinsically relativistic problem of neutral fermions subject to kink-like potentials $(\sim \tanh \gamma x)$ is investigated and the exact bound-state solutions are found. Apart from the lonely hump solutions for $E= \pm m c^{2}$, the problem is mapped into the exactly solvable Sturm-Liouville problem with a modified Pöschl-Teller potential. An apparent paradox concerning the uncertainty principle is solved by resorting to the concepts of effective mass and effective Compton wavelength.

(C) 2005 Elsevier B.V. All rights reserved.
\end{abstract}

\section{Introduction}

The four-dimensional Dirac equation with an anomalous magnetic-like (tensor) coupling describes the interaction of neutral fermions with electric fields and can be reduced to the two-dimensional Dirac equation with a pseudoscalar coupling when the fermion is limited to move in just one direction. Therefore, the investigation of the simpler Dirac equation in a $1+1$ dimension with a pseudoscalar potential might be relevant to a better understanding of the problem of neutral fermions subject to electric fields in the more realistic $3+1$ world.

The states of fermions in one-plus-one dimensions bound by a pseudoscalar double-step potential [1] and their scattering by a pseudoscalar step potential [2] have already been analyzed and some quite interesting results have been found. Indeed, the two-dimensional version of the anomalous magnetic-like interaction linear in the radial coordinate, christened by Moshinsky and Szczepaniak [3] as Dirac oscillator and extensively studied before [4-13], has also received attention. Nogami and Toyama [14], Toyama et al. [15] and Toyama and Nogami [16] studied the behaviour of wave packets under the influence of that parityconserving potential whereas Szmytkowski and Gruchowski [17] proved the completeness of the eigenfunctions. More re-

\footnotetext{
* Corresponding author.

E-mail address: hott@feg.unesp.br (M. Hott).
}

cently Pacheco et al. [18] studied a few thermodynamic properties of the $1+1$ dimensional Dirac oscillator, and a generalization of the Dirac oscillator for a negative coupling constant was presented in Ref. [19]. The two-dimensional generalized Dirac oscillator plus an inversely linear potential has also been addressed in Ref. [20].

In recent papers, Villalba [21] and McKeon and Van Leeuwen [22] considered a pseudoscalar Coulomb potential $(V=$ $\lambda / r)$ in $3+1$ dimensions and concluded that there are no bound states. The reason attributed in Ref. [22] for the absence of bound-state solutions is that the different parity eigenstates mix. Furthermore, the authors of Ref. [22] assert that the absence of bound states in this system confuses the role of the $\pi$-meson in the binding of nucleons. Such an intriguing conclusion sets the stage for the analysis by other sorts of pseudoscalar potentials. A natural question to ask is whether the absence of bound-state solutions by a pseudoscalar Coulomb potential is a characteristic feature of the four-dimensional world. In Ref. [19] the Dirac equation in one-plus-one dimensions with the pseudoscalar power-law potential $V=\mu|x|^{\delta}$ was approached and there it was concluded that $V$ is a binding potential only for $\delta>0$. That conclusion sharply contrasts with the result found in [22]. Ref. [19] shows that it is possible to find bound states for fermions interacting by a pseudoscalar potential in $1+1$ dimensions despite the fact that the spinor is not an eigenfunction of the parity operator. 
The parity-conserving pseudoscalar potential $\sim \tanh \gamma x$ is of interest in quantum field theory where topological classical backgrounds are responsible for inducing a fractional fermion number on the vacuum. Models of this kind, known as kink models, are obtained in quantum field theory as the continuum limit of linear polymer models [23-25]. To the best of our knowledge, no one has computed the complete set of bound states in the presence of this sort of potential. The present work investigates the bound-state solutions of fermions immersed in the background of the pseudoscalar potential $V=$ $\hbar c \gamma g \tanh \gamma x$, termed kink-like potential. A peculiar feature of this potential is the absence of bound states in a nonrelativistic theory because it gives rise to an ubiquitous repulsive potential. The whole spectrum of this intrinsically relativistic problem is found analytically, for both massive fermions and massless fermions. Fortunately, apart from solutions corresponding to $|E|=m c^{2}$, the problem is reducible to the finite set of solutions of the nonrelativistic exactly solvable symmetric modified Pöschl-Teller potential for both components of the Dirac spinor subject to a constraint on their nodal structure. Finally, we observe a remarkable feature of this problem: the possibility of trapping a fermion with an uncertainty in the position that can shrink without limit as $|\gamma|$ and $|g|$ increase without violating the Heisenberg uncertainty principle.

\section{The Dirac equation with a pseudoscalar potential in a $1+1$ dimension}

The $(1+1)$-dimensional time-independent Dirac equation for a fermion of rest mass $m$ coupled to a pseudoscalar potential reads

$H \psi=E \psi, \quad H=c \alpha p+\beta m c^{2}+\beta \gamma^{5} V$,

where $E$ is the energy of the fermion, $c$ is the velocity of light and $p$ is the momentum operator. The positive definite function $|\psi|^{2}=\psi^{\dagger} \psi$, satisfying a continuity equation, is interpreted as a position probability density and its norm is a constant of motion. This interpretation is completely satisfactory for singleparticle states [26]. We use $\alpha=\sigma_{1}$ and $\beta=\sigma_{3}$, where $\sigma_{1}$ and $\sigma_{3}$ are Pauli matrices, and $\beta \gamma^{5}=\sigma_{2}$. Provided that the spinor is written in terms of the upper and the lower components, $\psi_{+}$ and $\psi_{-}$respectively, the Dirac equation decomposes into:

$\left(-E \pm m c^{2}\right) \psi_{ \pm}=i \hbar c \psi_{\mp}^{\prime} \pm i V \psi_{\mp}$,

where the prime denotes differentiation with respect to $x$. In terms of $\psi_{+}$and $\psi_{-}$the spinor is normalized as

$$
\int_{-\infty}^{+\infty} d x\left(\left|\psi_{+}\right|^{2}+\left|\psi_{-}\right|^{2}\right)=1
$$

so that $\psi_{+}$and $\psi_{-}$are square integrable functions. It is clear from the pair of coupled first-order differential equations given by (2) that $\psi_{+}$and $\psi_{-}$have definite and opposite parities if the Dirac equation is covariant under $x \rightarrow-x$, i.e., if the pseudoscalar potential function is odd. The charge conjugation operation requires that if $\psi$ is a solution with eigenenergy $E$ for the potential $V$ then $\sigma_{1} \psi^{*}$ is a solution with eigenenergy $-E$ for the potential $-V$. It is interesting to note that the operation of just interchanging the upper and lower components of the Dirac spinor induced by $i \gamma^{5} \psi$ preserves the eigenenergies for a massless fermion when $V \rightarrow-V$. One can also see that the operator $\mathcal{O}=i\left[H, \sigma_{3}\right] / 2$ anticommutes with $H$ so that it maps positive- into negative-energy solutions, and vice versa. Although this last operator does not preserve the norm for scattering states, it can be used to obtain the normalized states corresponding to eigenenergies $-E$ from the knowledge of the normalized states with eigenenergies $E$.

In the nonrelativistic approximation (potential energies small compared to $m c^{2}$ and $E \approx m c^{2}$ ) Eq. (2) becomes

$$
\begin{aligned}
& \psi_{-}=\left(\frac{p}{2 m c}+i \frac{V}{2 m c^{2}}\right) \psi_{+}, \\
& \left(-\frac{\hbar^{2}}{2 m} \frac{d^{2}}{d x^{2}}+\frac{V^{2}}{2 m c^{2}}+\frac{\hbar V^{\prime}}{2 m c}\right) \psi_{+}=\left(E-m c^{2}\right) \psi_{+} .
\end{aligned}
$$

Eq. (3) shows that $\psi_{-}$is of order $v / c \ll 1$ relative to $\psi_{+}$ and Eq. (4) shows that $\psi_{+}$obeys the Schrödinger equation. Note that the pseudoscalar coupling has the effect that the Schrödinger equation has an effective potential in the nonrelativistic limit, and not the original potential itself. Indeed, this is the same side effect which in a $(3+1)$-dimensional space-time makes the tensor linear potential to manifest itself as a harmonic oscillator plus a strong spin-orbit coupling in the nonrelativistic limit [3]. The form in which the original potential appears in the effective potential, the $V^{2}$ term, allows us to infer that even a potential unbounded from below could be a confining potential. This phenomenon is inconceivable if one starts with the original potential in the nonrelativistic equation.

It should be noted that $V \rightarrow V+$ const in the Dirac equation and in its nonrelativistic limit does not yield $E \rightarrow E+$ const. Therefore, the potential and the energy themselves and not just the potential and energy differences have physical significance. It has already been verified that a constant added to the screened Coulomb potential [27] or to the inversely linear potential [28] is undoubtedly physically relevant. As a matter of fact, it plays a crucial role in ensuring the existence of bound states.

For $E \neq \pm m c^{2}$, the coupling between the upper and the lower components of the Dirac spinor can be formally eliminated when Eqs. (2) are written as second-order differential equations:

$-\frac{\hbar^{2}}{2} \psi_{ \pm}^{\prime \prime}+\left(\frac{V^{2}}{2 c^{2}} \pm \frac{\hbar}{2 c} V^{\prime}\right) \psi_{ \pm}=\frac{E^{2}-m^{2} c^{4}}{2 c^{2}} \psi_{ \pm}$.

This last result shows that the solution for this class of problem consists in searching for bound-state solutions for two Schrödinger equations. It should not be forgotten, though, that the equations for $\psi_{+}$or $\psi_{-}$are not indeed independent because $E$ appears in both equations. Therefore, one has to search for bound-state solutions for both signs in (5) with a common eigenvalue. At this stage one can realize that the Dirac energy levels are symmetrical about $E=0$. This means that the potential couples to the positive-energy component of the spinor in the same way it couples to the negative-energy component. 
In other words, this sort of potential couples to the mass of the fermion instead of its charge, so that there is no atmosphere for the spontaneous production of particle-antiparticle pairs. No matter what the intensity and sign of the potential is, the positive- and the negative-energy solutions never meet each other. Thus there is no room for transitions from positive- to negative-energy solutions. This all means that Klein's paradox never comes into the scenario.

The solutions for $E= \pm m c^{2}$, excluded from the SturmLiouville problem, can be obtained directly from the Dirac equation (2). One can observe that such isolated solutions, for $E=+m c^{2}$, are

$\psi_{-}=N_{-} \exp [-v(x)]$

$\psi_{+}^{\prime}-v^{\prime} \psi_{+}=+i \frac{2 m c}{\hbar} N_{-} \exp [-v(x)]$

and, for $E=-m c^{2}$,

$\psi_{+}=N_{+} \exp [+v(x)]$,

$\psi_{-}^{\prime}+v^{\prime} \psi_{-}=-i \frac{2 m c}{\hbar} N_{+} \exp [+v(x)]$,

where $N_{+}$and $N_{-}$are normalization constants and $v(x)=$ $\int^{x} d y V(y) /(\hbar c)$. Of course well-behaved eigenstates are possible only if $v(x)$ has an appropriate leading asymptotic behaviour.

\section{The kink-like potential}

Now let us concentrate our attention on the potential

$V=\hbar c \gamma g \tanh \gamma x$

where $\gamma$ and the dimensionless coupling constant, $g$, are real numbers. The potential is invariant under the change $\gamma \rightarrow-\gamma$ so that the results can depend only on $|\gamma|$ whereas the sign of $V$ depends on the sign of $g$. Since the solutions for different signs of $g$ can be connected by the charge conjugation transformation, and by the chiral transformation in the event of massless fermions, we restrict ourselves to the case $g>0$.

The Sturm-Liouville problem corresponding to Eq. (5) becomes

$-\frac{\hbar^{2}}{2 m_{\mathrm{eff}}} \psi_{ \pm}^{\prime \prime}+V_{\mathrm{eff}}^{[ \pm]} \psi_{ \pm}=E_{\mathrm{eff}} \psi_{ \pm}$

where we recognize the effective potential as the exactly solvable symmetric modified Pöschl-Teller potential [29-33] (in the notation of Refs. [31,32])

$V_{\mathrm{eff}}^{[ \pm]}(x)=-U_{0}^{[ \pm]} \operatorname{sech}^{2} \gamma x$

$U_{0}^{[ \pm]}=\frac{\hbar^{2} \gamma^{2}}{2 m_{\mathrm{eff}}} g(g \mp 1)>0 \quad \Rightarrow \quad g>1$

whose normalizable eigenfunctions corresponding to boundstate solutions, subject to the boundary conditions $\psi_{ \pm}=0$ as $|x| \rightarrow \infty$, are possible only if the effective potentials for both $\psi_{+}$and $\psi_{-}$present potential-well structures. According to (10), this demands that $g>1$. The corresponding effective eigenenergy is given by

$E_{\mathrm{eff}}=\frac{E^{2}-m_{\mathrm{eff}}^{2} c^{4}}{2 m_{\mathrm{eff}} c^{2}}=-\frac{\hbar^{2} \gamma^{2}}{2 m_{\mathrm{eff}}}\left(s_{ \pm}-n_{ \pm}\right)^{2}$,

where

$s_{ \pm}=\frac{1}{2}\left(-1+\sqrt{1+\frac{8 m_{\mathrm{eff}} U_{0}^{[ \pm]}}{\hbar^{2} \gamma^{2}}}\right) \Rightarrow\left\{\begin{array}{l}s_{+}=g-1, \\ s_{-}=g,\end{array}\right.$

$n_{ \pm}=0,1,2, \ldots<s_{ \pm}$

$m_{\mathrm{eff}}=\sqrt{m^{2}+\left(\frac{\hbar \gamma g}{c}\right)^{2}}$

Notice that $V_{\mathrm{eff}}^{[ \pm]}$is an even function under $x \rightarrow-x$. Furthermore, Eqs. (12) and (13) show that the capacity of the potential to hold bound-state solutions is independent of $\gamma$. As for $g$, it can be seen that the number of allowed bound states depends linearly on $g$ and there is always at least one bound-state solution for any $g>1$. From (10) and (11) one can note that the Dirac eigenenergies related to the bound-state solutions are restricted to the range

$\sqrt{m^{2} c^{4}+(\hbar c \gamma)^{2} g}<|E|<\sqrt{m^{2} c^{4}+(\hbar c \gamma)^{2} g^{2}}$

and that the eigenenergies in the range

$|E|>\sqrt{m^{2} c^{4}+(\hbar c \gamma)^{2} g^{2}}$

correspond to the continuum. Since the positive- and negativeeigenenergies never intercept each other, one can see once again that Klein's paradox is absent from this picture. In order to match the common effective eigenvalue for the effective potentials $V_{\mathrm{eff}}^{[+]}$and $V_{\mathrm{eff}}^{[-]}$one can see from (12) and (13) that the following constraint

$a_{n}=s_{+}-n_{+}=s_{-}-n_{-}=g-1-n_{+}$

must be satisfied. Eq. (16) implies that the quantum numbers $n_{+}$and $n_{-}$satisfy the relation

$n_{-}=n_{+}+1$.

This last fact can be better understood by observing that $V_{\mathrm{eff}}^{[+]}$ is deeper than $V_{\mathrm{eff}}^{[-]}$. Now, (11)-(14) tell us that

$E= \pm \sqrt{m^{2} c^{4}+(\hbar c \gamma)^{2}\left(g^{2}-a_{n}^{2}\right)}$

where

$n_{+}=0,1,2, \ldots<g-1$.

The upper and lower components of the Dirac spinor can be written as (see Ref. [32])

$$
\begin{aligned}
\psi_{ \pm}= & N_{ \pm} 2^{a_{n}} \Gamma\left(a_{n}+\frac{1}{2}\right) \sqrt{\frac{|\gamma| a_{n}}{\pi} \frac{\Gamma\left(n_{ \pm}+1\right)}{\Gamma\left(n_{ \pm}+1+2 a_{n}\right)}} \\
& \times\left(1-z^{2}\right)^{a_{n} / 2} C_{n_{ \pm}}^{\left(a_{n}+1 / 2\right)}(z),
\end{aligned}
$$

where $z=\tanh \gamma x$ and $C_{n}^{(a)}(z)$ is the Gegenbauer (ultraspherical) polynomial of degree $n$. Since $C_{n}^{(a)}(-z)=(-)^{n} C_{n}^{(a)}(z)$ 
and $C_{n}^{(a)}(z)$ has $n$ distinct zeros (see, e.g., [34]), it becomes clear that $\psi_{+}$and $\psi_{-}$have definite and opposite parities, as expected, and the nodes of $\psi_{+}$and $\psi_{-}$just differ by \pm 1 according to (17). The constants $N_{+}$and $N_{-}$are chosen such that $\int_{-\infty}^{+\infty} d x\left|\psi_{ \pm}\right|^{2}=\left|N_{ \pm}\right|^{2}$ and their absolute values can be determined by substituting (19) directly into the original first-order coupled equations (2) and demanding a Dirac spinor normalized to unity. By using a couple of recurrence relations involving the Gegenbauer polynomials (see, e.g., Ref. [34]) one can find that

$\left|N_{ \pm}\right|=\sqrt{\frac{E \pm m c^{2}}{2 E}}$.

Turning now to the isolated solutions, one can observe from (6) and (7) that a normalizable isolated solution is possible only if the upper component of the spinor vanishes and $E=-m c^{2}$. The normalized Dirac spinor can be written as

$\psi=\sqrt{\frac{|\gamma|}{\sqrt{\pi}} \frac{\Gamma(g+1 / 2)}{\Gamma(g)}}\left(1-z^{2}\right)^{g / 2}\left(\begin{array}{l}0 \\ 1\end{array}\right)$.

Note that the lonely hump probability amplitude does exist independently of the strength of $g$. One can also note that $\mathcal{O} \psi=0$ such that there is no state with $E=+m c^{2}$ (for $g>0$ ).

\section{Conclusions}

We have succeeded in obtaining the complete set of exact bound-state solutions of fermions in the background of a kinklike potential. Except for the solution $E=-m c^{2}$, the kink-like potential presents a spectral gap equal to

$2 \sqrt{m^{2} c^{4}+(\hbar c \gamma)^{2}(2 g-1)}$.

Since $C_{0}^{(a)}(z)=1$ (see, e.g., [34]) one can see that the position probability amplitude corresponding to the isolated solution given by (21) can be written in the very same mathematical structure of the remaining amplitudes. Thus, one could suspect that the isolated solution is just a particular case and that its existence is due to the particular method used in this Letter. However, the isolated solution has some distinctive characteristics when compared to the solutions of the Sturm-Liouville problem which lead us to believe that, in fact, they belong to a different class of solutions. The isolated solution breaks the symmetry of the energy levels about $E=0$ exhibited by the solutions of the Sturm-Liouville problem, and the corresponding eigenspinor has only one component differing from zero. It is this asymmetric spectral behaviour that leads to the fractionalization of the fermion number in quantum field theory [25]. Furthermore, unlike the Sturm-Liouville solutions, the isolated solution is there even if the kink-like potential is not so strong, i.e., there exists an isolated solution even if $g \leqslant 1$.

For massless fermions, except for $E=0$, the spectral gap equals to $2 \hbar c|\gamma| \sqrt{2 g-1}$ and the Dirac Hamiltonian anticommutes with $\sigma_{3}$ in such a way that the positive- and negativeeigenenergy solutions can be mapped by the operation $\psi_{-E}=$ $\sigma_{3} \psi_{E}$. The charge self-conjugate solution given by (21) appears now in the center of the spectral gap. As a matter of fact, the kink-like potential used for massless fermions as a solitonic scalar coupling [35] (of course one cannot distinguish a pseudoscalar from a scalar coupling for massless fermions) was used originally to show the generation of fractional fermion number from the charge self-conjugate solution.

It is noteworthy that the width of the position probability density for both class of solutions decreases as $|\gamma|$ or $g$ increases. As such it promises that the uncertainty in the position can shrink without limit. It seems that the uncertainty principle fails since such a principle implies that it is impossible to localize a particle in a region of space less than half of its Compton wavelength (see, for example, [36]). This apparent contradiction can be remedied by resorting to the concept of effective Compton wavelength defined as $\lambda_{\text {eff }}=\hbar /\left(m_{\text {eff }} c\right)$. Hence, the minimum uncertainty in the position consonant with the uncertainty principle is given by $\lambda_{\text {eff }} / 2$ whereas the maximum uncertainty in the momentum is given by $m_{\text {eff }} c$. It means that the localization of a neutral fermion under the influence of the kink-like potential can shrink to zero without spoiling the single-particle interpretation of the Dirac equation, even if the trapped neutral fermion is massless. It is true that as $|\gamma|$ or $g$ increases the binding potential becomes stronger, though, it contributes to increase the effective mass of the fermion in such a way that there is no energy available to produce fermionantifermion pairs.

As mentioned in the Introduction, the anomalous magneticlike coupling in the four-dimensional world turns into a pseudoscalar coupling in the two-dimensional world. The anomalous magnetic interaction has the form $-i \mu \beta \vec{\alpha} \cdot \vec{\nabla} \phi(r)$, where $\mu$ is the anomalous magnetic moment in units of the Bohr magneton and $\phi$ is the electric potential, i.e., the time component of a vector potential [26]. In one-plus-one dimensions the anomalous magnetic interaction turns into $\sigma_{2} \mu \phi^{\prime}$, then one might consider the kink potential as coming from an electric potential proportional to $\ln (\cosh \gamma x)^{g}$. Therefore, the problem addressed in this Letter could be considered as the one of trapping neutral fermions by a bowl-shaped electric potential.

\section{Acknowledgements}

The authors wish to thank an anonymous referee for very constructive remarks. This work was supported in part by means of funds provided by CNPq and FAPESP.

\section{References}

[1] A.S. de Castro, W.G. Pereira, Phys. Lett. A 308 (2003) 131.

[2] A.S. de Castro, Phys. Lett. A 309 (2003) 340.

[3] M. Moshinsky, A. Szczepaniak, J. Phys. A 22 (1989) L817.

[4] D. Itó, et al., Nuovo Cimento A 51 (1967) 1119.

[5] N.V.V. Swamy, Phys. Rev. 180 (1969) 1225.

[6] P.A. Cook, Lett. Nuovo Cimento 10 (1971) 419.

[7] E.F. Chaffin, J. Math. Phys. 14 (1973) 977.

[8] Y.M. Cho, Nuovo Cimento A 23 (1974) 550.

[9] H. Ui, G. Takeda, Prog. Theor. Phys. 72 (1984) 266.

[10] H. Ui, G. Takeda, Prog. Theor. Phys. 72 (1984) 813.

[11] A.B. Balanketin, Ann. Phys. (N.Y.) 164 (1985) 277.

[12] J.N. Ginocchio, in: B. Gruber, R. Lenczewski (Eds.), Symmetries in Science, vol. II, Plenum, New York, 1986. 
[13] R.J. Hughes, V.A. Kostelecky, M.M. Nieto, Phys. Rev. D 34 (1986) 1100.

[14] Y. Nogami, F.M. Toyama, Can. J. Phys. 74 (1996) 114.

[15] F.M. Toyama, et al., J. Phys. A 30 (1997) 2585.

[16] F.M. Toyama, Y. Nogami, Phys. Rev. A 59 (1999) 1056.

[17] R. Szmytkowski, M. Gruchowski, J. Phys. A 34 (2001) 4991.

[18] M.H. Pacheco, et al., Phys. Lett. A 311 (2003) 93.

[19] A.S. de Castro, Phys. Lett. A 318 (2003) 40.

[20] A.S. de Castro, Ann. Phys. (N.Y.) 311 (2004) 170.

[21] V.M. Villalba, Nuovo Cimento B 112 (1997) 109.

[22] D.G.C. McKeon, G. Van Leeuwen, Mod. Phys. Lett. A 17 (2002) 1961.

[23] J. Goldstone, F. Wilczek, Phys. Rev. Lett. 47 (1981) 986.

[24] R. Jackiw, G. Semenoff, Phys. Rev. Lett. 50 (1983) 439.

[25] For a review see: A.J. Niemi, G. Semenoff, Phys. Rep. 135 (1986) 99.

[26] B. Thaller, The Dirac Equation, Springer-Verlag, Berlin, 1992.
[27] A.S. de Castro, Ann. Phys. (N.Y.) 320 (2005) 56.

[28] A.S. de Castro, quant-ph/0510003, Int. J. Mod. Phys. A, in press.

[29] N. Rosen, P.M. Morse, Phys. Rev. 42 (1932) 210

[30] G. Pöschl, E. Teller, Z. Phys. 83 (1933) 143.

[31] L.D. Landau, E.M. Lifshitz, Quantum Mechanics, Pergamon, New York, 1958.

[32] M.M. Nieto, L.M. Simmons Jr., Phys. Rev. A 17 (1978) 1273.

[33] S. Flügge, Practical Quantum Mechanics, Springer-Verlag, Berlin, 1999.

[34] M. Abramowitz, I.A. Stegun, Handbook of Mathematical Functions, Dover, Toronto, 1965.

[35] R. Jackiw, C. Rebbi, Phys. Rev. D 13 (1976) 3398.

[36] P. Strange, Relativistic Quantum Mechanics, Cambridge Univ. Press, Cambridge, 1998. 